\title{
Desarrollo de la Biblioteca Digital Educativa EduCR@I 2.0
}

\author{
Yolanda López Santana \\ Alfredo Jonatan López Vargas \\ Centro Universitario de la Costa Sur de la Universidad de Guadalajara - México
}

CASE REPORTS

\begin{abstract}
Resumen
Las bibliotecas han evolucionado de ser lugares donde se almacenan, organizan y difunden la información hasta convertirse en centros dinámicos que actúan de manera proactiva en la construcción del conocimiento al disponer de recursos y servicios que innovan la manera de comunicarse con sus usuarios al generar espacios alternativos para lograr un impacto en la vida académica de la institución. La presente propuesta de biblioteca digital educativa consiste en la construcción de un espacio virtual extendido a la biblioteca física para albergar diferentes recursos con fines didácticos y educativos expresados en objetos de aprendizaje, canal de video, wikis, aula virtual, blogs, portal web, biblioteca en mundos virtuales y repositorios digitales. EDUCR@I 2.0 es el nombre que se le ha designado a la multiplataforma digital educativa de la biblioteca del CUCSUR, que presenta hasta el momento como avance en su construcción el diseño de estructuras para el desarrollo de competencias escritoras. El proyecto propone un esquema flexible al organizarse de forma modular y con posibilidades para incrementar sus componentes. Permite el ahorro de costos debido a la utilización de software libre y código abierto así como la intranet del campus y, dependiendo de la incorporación de materiales multimedia, usuarios conectados y documentos digitalizados a las bases de datos, es posible realizar pruebas preliminares de su funcionalidad con equipos de cómputo tipo Workstation existentes para la realización de pruebas preliminares y la medición de crecimiento de las colecciones a futuro.
\end{abstract}

Palabras clave

Biblioteca digital educativa; Blogs; Mediateca ; Biblioteca en mundos virtuales ; Software libre y código abierto ; Objetos de aprendizaje ; Web 2.0 ; Wikis ; Repositorios digitales ; Aula virtual

\section{Development of the educational digital library EduCR@I 2.0}

\begin{abstract}
Libraries have changed from being places where you store, organize and disseminate information, to become dynamic centers, acting proactively in knowledge construction by providing resources and services that innovate ways to communicate with its users to create alternative spaces to make an impact in the academic life of the institution. This educational digital library proposal involves the construction of a virtual space extended to the physical library resources to accommodate different teaching and educational purposes expressed in learning objects, video channel, wikis, virtual classroom, blogs, web portal, library in virtual worlds and digital repositories. EDUCR @ I 2.0 is the name that has been designated to the multi-platform educational digital library CUCSUR, presenting up to now as progress in its construction the design of structures for skills development writers. The project proposes a flexible schema to be organized in a modular way and the possibilities to increase its components. It allows saving costs due to the use of free software and open source as well as the campus intranet, and depending on the incorporation of multimedia materials, users online and scanned documents to database, it is possible to perform preliminary tests on its functionality with Workstations computer equipment type for preliminary testing and measuring growth of the collections in the future.
\end{abstract}

Keywords

Educational digital library ; Blogs ; Media center ; Library in virtual worlds ; Free software and open source ; Learning objects ; Web 2.0 ; Wikis ; Digital repositories ; Virtual classroom 


\section{Introducción}

La economía del conocimiento construye una redefinición de los valores otorgados a los bienes de producción. A diferencia de la producción de bienes tangibles en las pasadas etapas de la historia, los factores productivos como la tierra, el trabajo y el capital dan paso a nuevos modos de producir riqueza: el capital intelectual.

Hoy por hoy, el conocimiento es parte fundamental de la economía de los países. Al menos tres características vinculan al conocimiento con el desarrollo y la economía de los países: la globalización, proliferación de industrias y exportaciones de alta tecnología y el uso de las tecnologías de la información y la comunicación (TIC's) para la producción material y cultural.

Este hecho ha contribuido de manera importante para que se incluyan cambios en el rol del bibliotecario como un trabajador del conocimiento en la resolución de problemas de consulta, contribuyendo al principio del orden del universo de la información en expansión.

Esta nueva relación entre bibliotecas y usuarios hace necesario un replanteamiento de su perfil tradicional al convertirse en un ente dinámico, poseedor de competencias para la docencia, la investigación y el uso de aplicaciones informáticas, que permitan resolver de manera pragmática aspectos relacionados con el acceso eficiente a la información.

La adopción de modelos educativos centrados en el estudiante por las Instituciones de Educación Superior Públicas que buscan formar individuos con sentido crítico y espíritu investigativo para su posterior inserción a la sociedad productiva, exige de manera apremiante, el diseño de estrategias que respondan de forma eficiente al planteamiento de las necesidades de esta nueva economía.

Esta transformación también alcanza a las bibliotecas y las sitúa como los núcleos de la vida académica y focos de atracción para ofrecer diversos recursos informativos que apoyen al auto-aprendizaje, a pesar de las limitaciones económicas por las que se pueda estar pasando como institución, con propuestas innovadoras que salven dichas restricciones.

\section{La internet y las bibliotecas digitales educativas}

A partir de la creación de los hipertextos, la web se ha convertido en un espacio interactivo donde el usuario puede navegar en una lectura no lineal; en donde el usuario elige hacia dónde dirigirse y qué leer. De igual manera, las bibliotecas han evolucionado desde su función como espacio depositario, físico o virtual en donde se obtiene información para convertirse en un espacio educativo interactivo y formador, tomando un papel proactivo en la vida académica. Ese nuevo modo de comunicarse con sus usuarios ha generado, a partir de las bibliotecas híbridas, por conversión, las denominadas bibliotecas digitales educativas.

La captación de recursos educativos de información en este tipo de bibliotecas implica la concepción de dos tipos de competencias: la competencia de la lectura digital para recuperar y estructurar significativamente los recursos web y la competencia escritora, a través de los hipermedia por representación de contenidos.

La presente propuesta de biblioteca digital educativa consiste en la construcción de un espacio virtual extendido a la biblioteca física para albergar diferentes recursos con fines didácticos y educativos expresados en objetos de aprendizaje, canal de video, wikis, aula virtual, blogs, portal web, biblioteca en mundos virtuales y repositorios digitales. Cada uno de estos elementos pretenden contribuir al proceso de enseñanza-aprendizaje de los estudiantes en ambientes virtuales, lo que redundaría en un aprendizaje autogestivo o, también la opción de convertirse en material de apoyo para la enseñanza facilitada por el asesor en sesiones presenciales, utilizando la intranet del campus y la infraestructura física mediante conexión al cableado de fibra óptica multimodal entre los edificios de aulas y sus servidores, para proveer un eficiente flujo en la transferencia de datos.

EDUCR@। 2.0 es el nombre que se le ha designado a la multiplataforma digital educativa de la biblioteca del CUCSUR, que presenta hasta el momento, como avance en su construcción, el diseño de estructuras para el desarrollo de competencias escritoras.

El proyecto propone un esquema flexible al organizarse de forma modular $y$, con posibilidades para incrementar sus componentes. Permite el ahorro de costos, debido a la utilización de software libre y código abierto para cada uno 
de sus elementos y dependiendo de la incorporación de materiales multimedia, documentos digitalizados y usuarios conectados a las bases de datos. Esto es posible aseverarlo al haberse cubierto la etapa de diversas pruebas preliminares de funcionalidad con equipos de cómputo disponibles tipo Workstation, pero requerirá mejorarse a futuro debido al crecimiento de las colecciones.

Los ocho módulos iniciales de la multiplataforma son: Portal Web, Boletín Virtual, Biblioteca Digital, Biblioteca Virtual, Mediateca, Aula 2.0, Wikireferencias Bibliográficas y Objetos de Aprendizaje. La página concentradora de los módulos está disponible en el siguiente sitio: http://www.educrai.cucsur.udg.mx

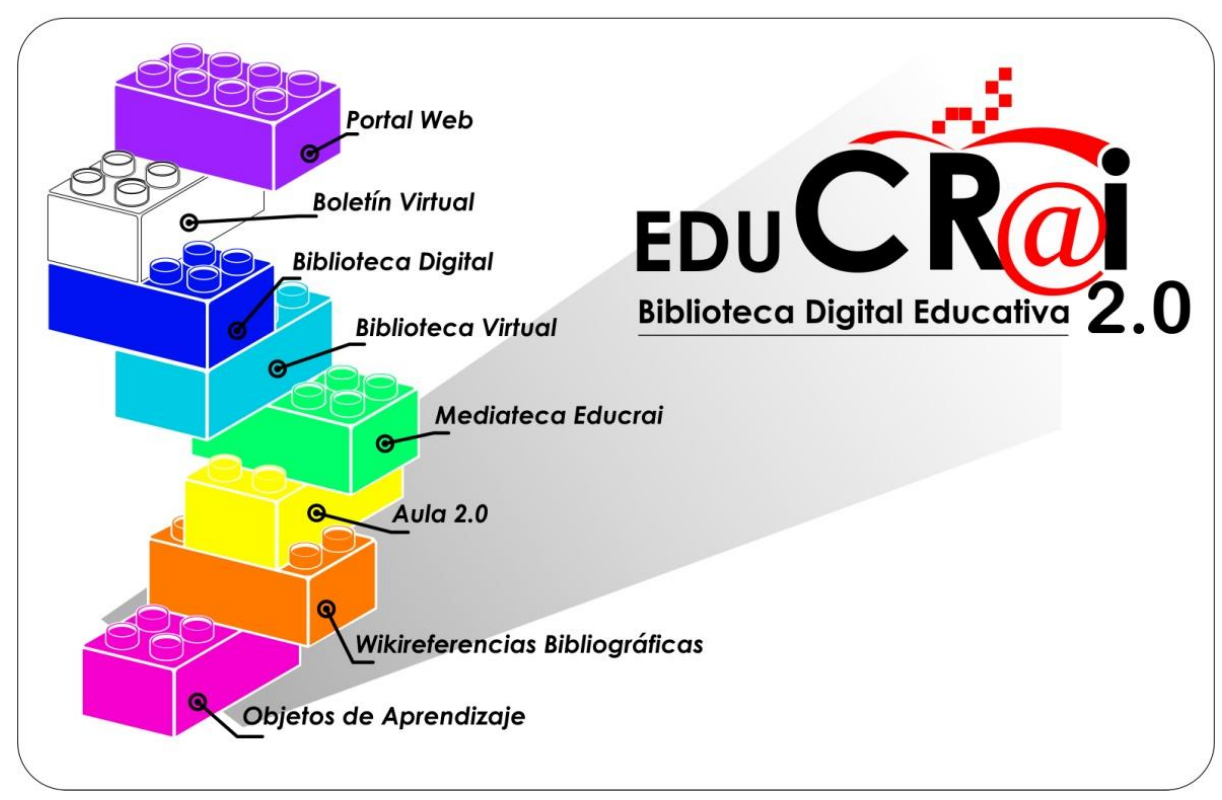

\section{Portal Web}

Toda interfaz de usuario deberá ser amigable y accesible con su universo de usuarios cautivos y potenciales, independientemente de sus capacidades visuales y motrices. Un sitio web accesible deberá ofrecer las mismas posibilidades de recibir sus contenidos y herramientas, tanto a un buscador de última generación como a uno obsoleto; así como a una persona que utiliza su mouse y a una que no puede hacerlo; tanto a un robot que viene a indexar sus páginas como a un invidente que la consulta mediante un intérprete auditivo.

Los usuarios de la web vienen con un objetivo específico: informarse, entretenerse, consultar o usar. Cualquiera que sea su objetivo, esperan conseguirlo sin que esto les represente un reto. Un sitio usable se preocupa porque los usuarios encuentren sus herramientas de la forma más natural e intuitiva sin necesidad de pensarlo, sin necesidad de averiguar dónde y cómo; las cosas simplemente deberán estar allí. Otro de los elementos indispensables de un portal web es la usabilidad del mismo para asegurar un flujo importante de usuarios a un sitio o portal.

Planear, diseñar o alfabetizar... cualquiera que sea nuestra actividad, un sitio web debe resolver un problema de investigación, informar sobre un acontecimiento, entretener o, simplemente encontrar un timón a la medida, con el cual navegar en el mar infinito de la información y una página web en bibliotecas, constituye uno de los principales medios de difusión de las actividades que contribuyen al desempeño académico de la comunidad a la que sirve.

El diseño del portal web EDUCR@I 2.0 sigue los lineamientos de homogeneidad de los portales de la Red Universidad de Guadalajara; fue desarrollado en Drupal, un sistema de gestión de contenidos que trabaja de manera modular para ser configurado por el usuario. Permite la publicación de textos, imágenes y otros servicios añadidos. El dominio electrónico designado por la Dirección General de Tecnologías de Información (DGTI) es: 


\section{Boletín virtual}

Un boletín de estas características permite la comunicación y retroalimentación con los usuarios en tiempo real, con opción del ahorro en la emisión de recursos impresos para la difusión de servicios, actividades y noticias a la comunidad a la cual es dirigido.

Inicialmente se analizan dos opciones para la creación del boletín virtual, en la primera de ellas se utilizó el Facebook; iniciativa que se dejó a un lado por el hecho de identificar que el usuario de esta plataforma, principalmente, busca permanecer en contacto con gente y hacer nuevos amigos, además de enterarse de las novedades de la comunidad a la que pertenece. La segunda opción se basó en el uso de un blog como un sitio para actualizar periódicamente noticias y/o eventos. De esta herramienta, se realizaron pruebas en dos de los más populares programas para su desarrollo: Blogger y WordPress.

Estos dos programas de software son gestores de contenido, que permiten crear páginas web muy fáciles de diseñar y sencillas para la incorporación de contenidos. La visualización de la información se presenta de manera cronológica permitiendo la administración de comentarios y la participación de varios autores. Estos gestores se caracterizan por facilitar la incorporación de widgets o gadgets, que consisten en pequeñas aplicaciones 0 programas que brindan accesorios para enriquecer el blog, como es el caso de calendarios, calculadoras, agendas, notas, entre otros recursos; demás de permitir la interacción con el usuario.

El boletín virtual EDUCR@I 2.0 fue desarrollado con WordPress. Consta de una sección de encabezado, incorpora seis menús desplegables: avisos al usuario, concursos y eventos, cursos, fomento a la lectura y módulos del proyecto EDUCR@I 2.0. Permite archivar mensualmente la bitácora de eventos publicados y favorece la interacción a través de comentarios. Requiere una base de datos en MySQL, lenguaje PHP y Servidor Apache. El acceso al mismo se realiza desde: http://148.202.114.5/wordpress/

\section{Biblioteca digital}

Las bibliotecas digitales son extensiones de la biblioteca física en donde se almacenan y distribuyen materiales bibliográficos que permitirán incrementar las opciones de consulta para el usuario de la información. Como su nombre lo indica, una biblioteca digital es aquella que cuenta con sus colecciones digitalizadas, es decir una representación de imágenes, textos o gráficos que pueden ser leídos con ayuda de un medio electrónico. A los espacios donde se guarda dicha información se les denomina repositorios. La disposición de los archivos puede ser a través del programa con el que fue generado el mismo, o bien, mediante conversión por aplicaciones lectoras que identifican la extensión -los tres caracteres posteriores al punto- del archivo.

La funcionalidad de una biblioteca digital radica en la organización de sus materiales para el préstamo o consulta y, el control de los documentos para realizar copia, impresión o descarga de los mismos. Las colecciones son organizadas a través de bases de datos que identifican los tipos de materiales y, su consulta se realiza mediante buscadores a través de diferentes puntos de acceso, los cuales son creados por los administradores del sistema y responden a la necesidad de sus usuarios para localizar recursos informativos a través del autor, tema, nombre de la publicación original, nombre del documento o tipo de recurso, entre otros.

Con la popularización de los diversos dispositivos de lectura electrónicos, los documentos digitales van ganando terreno para posicionarse como una nueva opción para la promoción de la lectura elevando el nivel cultural de la población. Las ventajas del formato digital permite, entre otras cosas, disminuir los costos de transportación, espacio de almacenaje y adquisición en línea del producto, acceso desde cualquier punto de la red sin necesidad de desplazamiento a la biblioteca, ahorro de tiempo en la búsqueda de la información a través de la búsqueda de contenido y accesibilidad mediante enlaces hipertextuales.

La biblioteca digital EDUCR@I 2.0 fue desarrollada en Greenstone, un conjunto de programas informáticos para la creación y difusión de colecciones documentales digitales. La plantilla de formato utilizada consta de una cintilla o banner principal, las colecciones creadas para proyectos de inversión, revistas, tesis y objetos de aprendizaje. Para su instalación se requiere un sistema cliente-servidor. Requiere base de datos en MySQL, plantillas PHP y servidor Apache. Este programa genera automáticamente sus módulos. 
El visualizador utilizado es la única excepción de uso de software propietario, el cual permite acceder al texto completo mediante tabla de contenidos, separadores, miniaturas de imágenes, visualización en pantalla completa y modificación de objetivos de distancia focal. Y el acceso al mismo se realiza desde la dirección: http://148.202.114.5:8080/greenstone/cgi-bin/library.cgi

\section{Biblioteca virtual}

Las bibliotecas virtuales generalmente son utilizadas como sinónimo de las bibliotecas digitales, sin embargo, para este contexto utilizamos una diferenciación importante e incluso, combinamos en algún momento ambos conceptos. La biblioteca virtual EDUCR@I 2.0 en mundos virtuales es una isla en el ciberespacio. Está diseñada para trabajar interactuando con el usuario, el cual podrá sumergirse en la plataforma a través de su ávatar, es decir, una cibercriatura que puede sentarse, caminar, correr, volar y teletransportarse. Los avatares generalmente se podrán visualizar dando la espalda, sus movimientos son controlados por el mismo usuario; permite comunicación mediante chat o voz; puede transformar su apariencia con mucha flexibilidad, usar gestos, poses y actitudes que lo identifican además de variar su vestuario elegido desde un inventario disponible.

El software utilizado para la construcción del escenario se realizó en OpenSim; una multiplataforma de código abierto que permite desarrollar un entorno virtual propio. Es una plataforma multiusuario similar a Second Life donde el usuario podrá socializar, explorar, descubrir y poner a prueba su creatividad en un entorno 3D.

Los mundos virtuales se caracterizan por permitir un espacio compartido, el uso de interfaces gráficas, interacción en tiempo real, contenidos generados por los usuarios y un soporte activo por grupos especializados a través de las redes sociales.

Para una interacción entre el Servidor y el equipo cliente, se recomienda un fluido ancho de banda de 100 Megabytes, en salida de datos y un visor como Second Life, Thunderbird o Phoenix viewer. El acceso se realiza desde el sitio web: http://148.202.114.89/bibliotecavirtual/index.html

El módulo de Biblioteca Virtual se encuentra integrado con base en tres servicios iniciales: referencia virtual, auditorio de videoconferencias virtuales y la sala de exposiciones fotográficas y pictóricas.

Referencia virtual: es el área de referencia virtual donde podrá tener acceso directo al catálogo electrónico WebOpac; de igual manera, un acceso directo a la Biblioteca Digital WdgBiblio de la Red de Bibliotecas de la Universidad de Guadalajara -es en este espacio donde convergen la biblioteca virtual y la digital-. En este mismo sitio se podrá interactuar con el avatar bibliotecario que atenderá las solicitudes de orientación y búsqueda de información.

Auditorio y sala de videoconferencias: son dos espacios virtuales diseñados para obtener video y audio streaming, es decir, permite visualizar y tener acceso auditivo a un archivo mientras éste está siendo descargado de un servidor para ser reproducido en la computadora del cliente, al igual que la utilización de tecnologías para el enlace de dos puntos ubicados en espacios geográficos distantes y comunicados en tiempo real para la proyección de videoconferencias.

El área exposiciones: es el espacio más amplio de la plataforma, acondicionado con un edificio para contener la pinacoteca "José Atanasio Monroy" del CUCSUR. El edificio de la pinacoteca consta de dos pisos, en la planta baja encontrará la colección "José Atanasio Monroy", compuesta por obras adquiridas por el CUCSUR al pintor. En el segundo piso se exponen las obras ganadoras del Premio Nacional de Pintura "José Atanasio Monroy".

En un área exterior al edificio, cercana al espacio virtual de referencia, se encuentra la segunda área de exposiciones con una muestra fotográfica de la "Sierra de Manantlán".

\section{Mediateca}

Los recursos multimedia son parte fundamental en el aprendizaje del individuo de la era digital. Las bibliotecas de hoy incorporan cada vez más, recursos y espacios destinados a este tipo de colecciones. Las mediatecas albergan las colecciones magnético-digitales, e incorporan un mayor número de equipos de cómputo para el acceso en línea.

Los materiales didácticos y recursos educativos multimedia, disponibles en sitios web educativos y en los repositorios institucionales, son alternativas de interés para quienes buscan información a través de estos sistemas. 
En la actualidad son utilizados como medio complementario de los textos para la comprensión del tema y el trabajo extendido en el aula, pero por las características propias de los recursos, responden a las demandas que genera un proceso constructivista del conocimiento.

La mediateca virtual EDUCR@I 2.0 permite acceso a recursos multimedia (imágenes, video y audio) para fortalecer el aprendizaje autónomo del estudiante. Consta de una cintilla principal como encabezado, barra de menús y campo de búsqueda por tema. Presentación de videos por categoría del más reciente, el más popular y los más votados.

La plataforma se realizó en Joomla, un gestor de contenidos de código abierto que posee un gran número de extensiones para enriquecer los resultados del proyecto, mismas que son grupadas en: componentes, módulos, plantillas y complementos o plugins que permiten la incorporación de aplicaciones. Para su instalación requiere un servidor Apache, una base de datos MySQL y un intérprete de lenguaje PHP preferentemente. El acceso se realiza a través del punto: http://148.202.114.14/

\section{Aula 2.0}

Los nuevos paradigmas educativos contemplan los actuales escenarios y se formula el concepto de aula extendida para denominar a los espacios virtuales, en donde las comunidades aprendientes son organizadas por los propios individuos que las conforman, interactuando y comunicándose por medios electrónicos.

El contexto educativo derivado del uso de las TIC's justifica una eficiente gestión de contenidos apoyados en ambientes educativos virtuales, que permitan un aprendizaje centrado en el estudiante, colaborativo y con independencia en los horarios de trabajo, con facilidades de acceso a los sistemas de evaluaciones del aprendizaje; control de los tiempos de entregas de actividades; acceso a archivos digitales, multimedios y conexión a sitios web; procedimientos formales de evaluación; comunicación vía correo electrónico, foros y mensajería instantánea en apoyo al aprendizaje, entre otros aspectos.

El aprendizaje electrónico también conocido como e-learning caracteriza un estudiante "en línea" que puede comunicarse con sus "compañeros de grupo" y sus "tutores" de manera síncrona o asíncrona sin una limitación geográfica, laboral, de edades, sexo o diferencias ideológicas, lo que enriquece de manera significativa el aprendizaje colectivo, extendiendo las posibilidades de formación a un mayor número de personas.

Aula EDUCR@I 2.0 fue diseñada en Moodle, un sistema de gestión de cursos de distribución libre, cuya plataforma es muy flexible para el diseño instruccional en modalidad presencial, semipresencial y virtual. El programa de software permite acceder a diversos módulos para control de tareas, consultas, foros, cuestionarios, recursos didácticos, encuestas y estadísticas. Para su instalación requiere MySQL, lenguaje de programación PHP y servidor Apache. El acceso a la plataforma se puede direccionar desde: http://148.202.114.89/moodle/moodle/

\section{Wikireferencias bibliográficas}

La transición de la Web 1.0 a la Web social o Web 2.0 implica que nuevos espacios continúan produciéndose en el entramado. Ambas redes cumplen las funciones para las cuales fueron creadas; la primera de ellas es una red receptiva e informativa que entrega información a sus usuarios; mientras tanto la web 2.0 es una red en donde sus usuarios dialogan e interactúan entre sí y se apuesta a la confianza para una edición colectiva.

Una de las herramientas que pueden ser utilizadas en ambientes virtuales colaborativos son los wikis. Un wiki permite la posibilidad de escribir y editar documentos de forma colectiva. Su actualización es inmediata y registra un control de cambios al generar un archivo de adiciones y modificaciones. Son verdaderos medios de hipertexto al generar una estructura de navegación multinivel mediante lecturas asociativas.

Para el presente módulo se utiliza software MediaWiki, es un sistema gestor de contenidos con licencia GNU, es decir, una licencia pública general para usar, distribuir y modificar el software. El sistema soporta múltiples usuarios y diversos niveles de acceso, plantillas para modificar apariencia y la incorporación de imágenes. Posee herramientas para obtención de información y diversos controles para enterarse sobre los cambios realizados al proyecto. Requiere MySQL, servidor Apache y lenguaje PHP. 
El módulo de Wikireferencias bibliográficas de EDUCR@I 2.0 fue diseñado para trabajar colaborativamente en una estructura establecida, añadiendo de acuerdo al programa educativo y carrera correspondiente la bibliografía básica, complementaria y recomendada por los co-autores del wiki con la finalidad de aumentar las opciones de consulta a las fuentes de información para sus asignaturas. El acceso a la wikibibliografía se puede obtener desde: http://148.202.114.89/mediawiki/index.php/P\%C3\%A1gina principal

\section{Objetos de aprendizaje}

Los objetos de aprendizaje (OA), son entidades digitales o no digitales con propósito pedagógico orientado al modelo constructivista. Están compuestos por unidades de información auto-contenida que puede ser reutilizada para apoyar el aprendizaje mediante contenidos, actividades y evaluaciones sobre una temática. Los OA utilizan catalogación con base en estándares internacionales, con la finalidad de facilitar el intercambio e interoperabilidad con diversos repositorios y plataformas.

El desarrollo de un OA implica, entre otras cosas, la participación colaborativa de especialistas en la materia, pedagogos, bibliotecarios, diseñadores de hipermedia, diseño gráfico y del área informática. Cada uno de ellos resolverá en su momento los requerimientos del producto final, dirigidos por el experto en la materia quien se encargará también de la difusión e innovación didáctica para su utilización en la enseñanza.

La estructura del OA generado en la plataforma EDUCR@I 2.0 fue desarrollado en eXe, una herramienta de código abierto creada para facilitar la construcción de contenidos e-learning. Este software permite la incorporación de variados elementos como son textos, videos, audio e imágenes en acceso local o con enlace desde de la web. El programa también admite la creación de módulos para enriquecer el OA, tales como artículos Wiki, sindicación de contenidos RSS, videos desde Youtube y entornos para la creación, realización y evaluación de actividades multimedia con JClic o bien, la exportación del producto a la plataforma Moodle mediante un archivo SCORM. Requiere servidor Apache para su publicación en la web. El acceso al repositorio de objetos de aprendizaje se puede realizar con el acceso a la plataforma de la biblioteca digital disponible en: http://148.202.114.5:8080/greenstone/cgi-bin/library.cgi

\section{Conclusiones}

El modelo de biblioteca digital educativa cobra significativa importancia en el contexto espacial concebido hasta ahora, al implicar un replanteamiento de los entornos bibliotecarios tradicionales, además del rediseño de nuevas áreas para albergar equipos de cómputo con acceso a internet, laboratorio de idiomas y multimedia, área de facilidades tecnológicas para la edición, impresión, producción y post-producción; espacios para trabajo en equipo, áreas culturales y recreativas así como un replanteamiento de los servicios tradicionales mediante la instrumentación de catálogos automatizados, depósito de materiales docentes, repositorios de objetos de aprendizaje, depósitos de archivos gráficos (videos y multimedios), depósitos de libros electrónicos y revistas electrónicas, servicio de préstamo e información en línea, organización de la información electrónica producida por la institución y servicio de soporte técnico y asesoramiento tecnológico.

La aplicación de programas de software de libre acceso y código abierto permiten el desarrollo de plataformas, para la creación de colecciones digitales y recursos informativos virtuales en instituciones de educación con limitados recursos económicos, para facilitar el aprendizaje y la retroalimentación con sus estudiantes y así fomentar el trabajo colaborativo y la conformación de comunidades aprendientes que comparten, descubren y disfrutan vivir en el ciberespacio, el principal medio de comunicación para la sociedad del conocimiento. 


\section{Bibliografía}

Bernardez, M. (2008). Capital Intelectual. Creación de valor en la sociedad del conocimiento. [en línea] Consultado el 18 Enero del 2012. Disponible

http://books.google.com.mx/books?hl=es\&lr=\&id=pFBxQOvMRswC\&oi=fnd\&pg=PA17\&dq=\%22trabajador+del+conocimiento\%22+ AND+bibliotecas\&ots=7cW2iXNZE2\&siq=i81a7npOQ9pv5vbXUfoDp99vNXo\#v=0nepage\&q=\%22trabajador\%20del\%20conocimient $0 \% 22 \% 20$ AND $\% 20$ bibliotecas\& $f=$ false

Cataldi, Z. (2005). Evaluación de programas hipermedia educativos de producto final y en un contexto similar al de aplicación. [en línea] Consultado el 24 de agosto de 2010. Disponible en: http://www.unex.es/didactica/RELATEC/sumario 4 2.htm

Djorgovski, S.G. \& Curius, G. (2011). Getting started in Second Life and OpenSim. [en línea] Recuperado el día 06 de enero de 2012. Disponible en: http://dame.dsf.unina.it/astroinformatics/lectures/GettingStartedinSL.pdf

Kobak, A. \& Ozarslan, Y. (2011). Views of students about the use of Second Life for educational purpose. [en línea] Recuperado el día 06 de enero de 2012. Disponible en: https://tojde.anadolu.edu.tr/tojde43-2/pdf/article 9.pdf

López, C. (2005 ). Los repositorios de objetos de aprendizaje como soporte para los entornos e-learning. [en línea] Consultado el 26 de Agosto de 2010. Disponible en: http://www.biblioweb.dgsca.unam.mx/libros/repositorios/objetos aprendizaje.htm.

Martínez, S.; Bonet, P.; Cáceres, P.; Fargueta, F.; García, E. (2007). Los objetos de aprendizaje como recurso de calidad para la docencia: criterios de validación de objetos en la Universidad Politécnica de Valencia. [en línea] Consultado el 06 de Septiembre de 2010. Disponible en: http://spdece07.ehu.es/actas/Naharro.pdf

Marzal, M.A. (2008). La alfabetización en información como dimensión de un nuevo modelo educativo: la innovación docente desde la documentación y los CRAI. [en línea]. Recuperado en mayo 18 de julio de 2010. Disponible en: http://dialnet.unirioja.es/servlet/articulo?codigo=2730304

Marzal, M.A. (2008). La irresistible ascensión del CRAl en la universidad. [en línea]. Recuperado el 20 de julio de 2010. Disponible en: http://www.portalseer.ufba.br/index.php/revistaici/article/view/2667/1883

Marzal, M.A., Calzada-Prado, J. \& Vianello, M. (2008). Criterios para la evaluación de la usabilidad de los recursos educativos virtuales: un análisis desde la alfabetización en información. [en línea] Recuperado el día 18 de mayo de 2010. Disponible en: http://informationr.net/ir/13-4/paper387.html

McKelvey, F. (2001). A Programmable Platform? Drupal, Modularity, and the Future of the Web. [en línea] Recuperado el día 06 de enero de 2012. Disponible en: http://fibreculturejournal.org/wp-content/pdfs/FCJ-128Fenwick\%20McKelvey.pdf

Patel, S.K., Rathod, V.R. \& Prajapati, J.B. (2011). Performance Analysis of Content Management Systems Joomla, Drupal and WordPress. [en línea] Recuperado el día 06 de enero de $2012 . \quad$ Disponible en: http://www.ijcaonline.org/volume21/number4/pxc3873373.pdf

Reinoso, J.A. (2010). Análisis de la incorporación de una plataforma wiki a la docencia de la asignatura "nuevas tecnologías de la información" [en línea] Recuperado el día 06 de enero de 2012. Disponible en: http://redalyc. uaemex.mx/src/inicio/ArtPdfRed.jsp?iCve=54714042006

Sanchez, G. (Ed). (2009). América Latina y el Caribe en la Economía y Sociedad del Conocimiento. Una revisión crítica a sus fundamentos y políticas. [en línea]. Consultado el 18 Enero del 2012. Disponible en: http://bibliotecavirtual.clacso.org.ar/ar/libros/campus/dazacono.pdf\#page=46

Scagnoli, N. (2005). Estrategias para motivar el aprendizaje colaborativo en cursos a distancia. [en línea]. Recuperado el 17 de junio de 2010. Disponible en: http://www.ideals.uiuc.edu/bitstream/handle/2142/10681/aprendizaje-colaborativoscagnoli.pdf? sequence=2 


\section{Datos de los autores}

\section{Yolanda López Santana}

Técnico académico asociado B. Titular de la Plataforma EduCR@I 2.0. Egresada de la Maestría en Ciencias de la Información y Administración del Conocimiento de la Universidad Virtual del ITESM. Actualmente realiza Actividades de Desarrollo de Habilidades Informativas en el Centro Universitario de la Costa Sur de la Universidad de Guadalajara y es profesora de asignatura de la Universidad Virtual.

ysantana@cucsur.udg.mx

\section{Alfredo Jonatan López Vargas}

Egresado de la Maestría en Gestión de Tecnologías de Información de la Universidad Tec Milenio. Soporte Técnico de la Biblioteca Antonio Alatorre Chavez del Centro Universitario de la Costa Sur de la Universidad de Guadalajara. Profesor de asignatura del CUCSUR y Administrador de Multiplataforma del proyecto Educr@i 2.0.

jonatan.lopez@cucsur.udg.mx

Recibido-Received : 2012-06-19

Aceptado-Accepted : 2012-09-29

\section{(cc) BY-NC-ND}

This work is licensed under a Creative Commons

Attribution-Noncommercial-No Derivative Works 3.0 United States License.

\section{ULIS D-Sonte}

This journal is published by the University Library System of the University of Pittsburgh as part of its $\underline{D-S c r i b e}$ Digital Publishing Program and is cosponsored by the University of Pittsburgh Press. 